\title{
Functional MRI in Depression-Multivariate Analysis of Emotional Task
}

\author{
Sevdalina Kandilarova ${ }^{1,2} \cdot$ Drozdstoy Stoyanov $^{1,2} \cdot$ Magdalena Stoeva $^{3} \cdot$ Adeliya Latypova $^{4} \cdot$ Ferath Kherif $^{4}$
}

Received: 12 May 2020 / Accepted: 24 June 2020 / Published online: 7 July 2020

(c) Taiwanese Society of Biomedical Engineering 2020

\begin{abstract}
Rationale, Aims and Objectives The field of translational neuroscience suffers from an extremely low replication levels compared to other life science fields. The objective of the present study was to test the hypothesis that multivariate analysis of a classical emotional pictures paradigm would produce meaningful brain signatures with some power to discriminate depressed patients from healthy subjects.

Methods Participants in the study were eighteen medicated depressed patients and eighteen sex and age matched healthy controls. Functional MRI paradigm with a visual presentation of emotional pictures (positive, negative and neutral) from the International Affective Pictures System was used. The multivariate linear method (MLM) was used to derive the specific brain signatures on an individual and on a group level. The predictive power of the brain signatures is tested by use of linear discriminant analysis.

Results Following the individual and group MLM, the three brain patterns that summarized all the individual variabilities of the individual brain patterns were produced. The discriminant analysis yielded accuracy levels for the three brain signatures ranging from 67 to $98 \%$.

Conclusion The present study demonstrated that the multivariate linear method resulted in meaningful brain signatures with significant potential for distinction between healthy and depressed subjects. Such findings will fuel the emerging paradigm shift from more conventional statistical analysis to the probably more appropriate for the field of functional neuroimaging machine learning techniques.
\end{abstract}

Keywords Functional magnetic resonance imaging $\cdot$ Multivariate analysis $\cdot$ Depression $\cdot$ Affective pictures $\cdot$ Clinical diagnosis

\section{Introduction}

Depression is considered major psychiatric disorder of social burden, anticipated second most common disease worldwide by 2030 [1]. The manifestations of depression include

Magdalena Stoeva

magdalena.stoeva@mu-plovdiv.bg

1 Department of Psychiatry and Medical Psychology, Medical University of Plovdiv, Plovdiv, Bulgaria

2 Research Institute at Medical University of Plovdiv (RIMU), Plovdiv, Bulgaria

3 Translational Neurosciences Centre, Medical University of Plovdiv, Plovdiv, Bulgaria

4 Faculté de Biologie Et de médecine, Laboratoire de Recherche en Neuroimagerie (LREN), Université de Lausanne, Lausanne, Switzerland affective, cognitive and somatic symptoms, determined in common clinical practice with self-evaluation and observational inventories. Advanced cognitive and computational neuroscience has provided important insights into the biological mechanisms of depressive disorders [2], which nonetheless have been found to be difficult to incorporate into the dominating diagnostic systems. This is due to a great extent to the inconsistencies in the study designs and relevant findings as well as the limitations for meaningful translation of data from neuroscience to psychiatry [3].

The mainstream hypothesis is that depressed subjects demonstrate negative bias during processing affective e.g. emotional stimuli [4] explained by increased reactivity of the amygdala to negative stimuli typically associated with decreased activity of the prefrontal cortex in depression as compared to healthy population. This pattern is reversed after successful antidepressant treatment [5-9]. More 
specifically investigations performed with International Affective Picture System (IAPS) paradigm produce results about increased activation of amygdala, insula and parahippocampal area in depressed patients as compared to healthy controls during processing of negative vs neutral emotional stimuli [5]. Such hypothesis was challenged by investigations of patients which yielded activation in prefrontal cortex during viewing negative vs neutral pictures without any difference in amygdala response [10].

In line with many influential publications in the field of neuroimaging the above are based on either pre-defined ROIs implicated in emotion processing from previous research and rather liberal statistical significance threshold of $\mathrm{p}<0.005$ uncorrected for multiple comparisons, which are two of the major confounds considered to undermine the recent efforts to translate neuroimaging findings into clinical practice $[11,12]$. On the level of meta-analyses there has been summarized initially encouraging convergence of the individual study results $[13,14]$, yet the most recent ones have failed to replicate those findings $[15,16]$, which boosts the debate about replication crisis in the field [17, 18].

In particular those controversies are interpreted in terms of heterogeneity of inclusion/exclusion criteria for construction of patient samples (especially depression severity remission, medication status), variability across research centres and networks of the paradigms and wide range of the statistical analyses performed. In order to address those caveats, there has been stressed the need of replication and application of robust statistical methods [12, 19].

In our previous research [20], an actual precursor of the current paper we have attempted to replicate the findings of altered emotional processing in depressed patients compared to healthy controls by means of functional magnetic resonance imaging (fMRI) during passive viewing of positive, negative and neutral (Pos, Neg and Neu) pictures from the International Affective Pictures System (IAPS) [21]. The whole brain voxel-wise analysis with the most stringent, family wise error $(\mathrm{FWE})$ correction $(\mathrm{p}<0.05)$ did not yield significant between group differences.

In the present study we analyze the same sample by use of machine learning technique, multivariate linear method. It was driven by the results achieved by similar methodological frameshift applied to another, innovative paradigm, comprised of the items from clinical self-evaluation scale for depression. In this paradigm Paranoid-Depressive Scale of Von Zerssen was administered simultaneously with the functional MRI BOLD (Blood Oxygenation Level Dependent) signal acquisition. The conventional mass univariate analysis did not cross the significance threshold on between-group level after inclusion of gender as co-variate and correction for multiple comparisons (FEW p <0.05) in standard two sample t-test analysis procedure [3, 22]. When we applied multivariate linear method (MLM) to the same sample, the brain signatures behind each group of item responses (paranoid, depressive, and neutral) underpinned strong discriminative power for respective psychiatric disorders, major depressive disorder and schizophrenia [23].

In that respect we have decided to employ alternative approach of cross-validation of IAPS and brain activation patterns by adopting the machine learning methodology. The hallmark of our rationale is to experimentally confirm the theory of translational cross-validation across neuroscience and psychiatry as defined elsewhere [24-26]. According to this theory convergence between psychopathological and functional MRI measures may be achieved in terms of synchronization of data acquisition, i.e. simultaneous application of the two methods. This may facilitate the translation from the clinical psychopathology domain to neuroimaging and vice versa.

\section{Aim}

The objective of the present study was to test the hypothesis that the characteristic distribution of regional BOLD signal changes captured through MLM where the brain signatures identified by three principal components based on activations yielded from the three kinds of emotional stimuli (Pos, $\mathrm{Neg}$ and $\mathrm{Neu}$ ) in depressed patients would have some power to discriminate them from healthy subjects.

\section{Methods}

\subsection{Subjects}

This study includes total 36 right-handed adult subjects divided into 2 groups and matched by age and sex between the groups. The distribution of the subjects follows the below pattern:

- 18 adult subjects (mean age $44.5 \pm 13.6$ y, 5/13 males/ females) suffering from a depressive episode (single or recurrent, according to the DSM-IV-TR criteria) in the context of major depressive disorder (MDD, 12 participants) or bipolar affective disorder (BD, 6 participants)

- 18 healthy controls (mean age 38.7 \pm 13.7 y, 5/13 males/ females).

All patients were medicated with various antidepressants and mood stabilizers (including escitalopram, sertraline, venlafaxine, duloxetine, lamotrigine, olanzapine) for at least 3 weeks before enrolment. 


\subsection{Pre-MRI Stage}

The fMRI testing is preceded by 3-component clinical evaluation-general psychiatric interview; structured Mini International Neuropsychiatric Interview-M.I.N.I 6.0 [27] and Montgomery-Åsberg Depression Rating Scale_-MADRS [28].

\subsubsection{Inclusion Criteria}

Total score of at least 20 points on MADRS for depressed individuals; lack of psychiatric history, major neurological or somatic illness affecting the CNS, head traumas causing loss of consciousness for healthy controls.

\subsubsection{Exclusion Criteria}

Presence of second axis I diagnosis (any of but not limited to psychotic, anxiety, substance related disorder), severe decompensated somatic illness, neurological disease, history of head trauma with loss of consciousness or severe suicidal risk (10th item of MADRS $\geq 2$ ).

\subsubsection{Ethics}

Informed consent complying with the Declaration of Helsinki is officially signed by all participants. This study is approved by the University's Ethics Committee.

\subsection{MRI Data Acquisition}

\subsubsection{Equipment and Tools}

3 T MRI system (GE Discovery 750w) was used as a main data acquisition tool, in combination with goggles (Visual System, NordicNeuroLab, Bergen, Norway) to project the visual stimuli to the individuals.

\subsubsection{Scanning Protocol}

The scanning protocol is a combination between HiRes anatomical scan and functional scan to ensure full brain coverage and obtain best structural and functional datasets. Anatomical scan parameters-Sag 3D T1 FSPGR, slice thickness $1 \mathrm{~mm}$, matrix $256 \times 256, \mathrm{TR}=7.2 \mathrm{~ms}, \mathrm{TE}=2.3$, flip angle $12^{\circ}$. Functional scan parameters-2D EPI, slice thickness $3 \mathrm{~mm}$, spacing $0.5,36$ axial slices, matrix $64 \times 64$, $\mathrm{TR}=2000 \mathrm{~ms}, \mathrm{TE}=30 \mathrm{~ms}$, flip angle $90^{\circ}, 240$ volumes.

\section{4 fMRI Design}

The functional paradigm was designed and presented via E-prime software (Psychology Software Tools, Inc). IAPS served as the main source for images equally distributed (20 each) between Positive (Pos), Negative (Neg), and Neutral (Neu). The selection criterion is based on valence scores within the ranges 7.04-8.2, 1.8-2.49, and 4.38-5.22 respectively. The homogeneity of the stimuli within each group is ensured by a proper selection of images presenting humans at different ages and under similar proportions.

The functional paradigm consists of sequences of 12 off-blocks and 12 (4 of each type) active blocks, each with duration of $20 \mathrm{~s}$. While the off-blocks only presented a fixation cross on monochrome background, the active blocks contained sequences of 5 images with equal duration $(4 \mathrm{~s})$ within the block.

A set of 20 feedback controls were additionally introduced to ensure the subjects attended the stimuli. The controls registered the subject's attention by presenting equally distributed elements (red dots) superimposed on the image projected during the off-blocks and the active blocks, and demanding an action (pressing a button) by the subject.

\section{5 fMRI Data Analysis}

fMRI data analysis was performed using the SPM 12 software package (Statistical Paramertic Mapping, https:// www.fil.ion.ucl.ac.uk/spm/) in combination with MATLAB R2020 running on MS Windows environment. Data analysis comprises of two main stages-preprocessing and first-level analysis.

Preprocessing included realigning the fMRI images to the first image (motion correction), which were then coregistered with the high-resolution structural images, normalized to Montreal Neurological Institute (MNI) space, and smoothed with a $6 \mathrm{~mm}$ full-width-at-half-maximum Gaussian kernel.

First-level analysis of the fMRI (BOLD) responses was conducted using a general linear model (GLM) as a major tool recognized for fMRI data analysis. GLM was applied to the time series, convolved with a canonical hemodynamic response function. Nuisance covariates included the six rigid body motion parameters. F-contrast of all three active conditions vs the off condition was calculated for each individual dataset.

\subsection{Multivariate Analysis}

MLM is applied on the highly-dimensional data to explain the variance of the data in its distinct features with minimal loss of information. This method is characterized by three core advantages. Opposed to similar dimension reduction methods such as principal component analysis (PCA), MLM takes into account information coming not only from the data $(Y)$, but also form the design matrix $(X)$, e.g. contextual, experimental, behavioural. In addition, 
MLM is specially adapted to fMRI data by including in the model the temporal autocorrelation from the noise. Thus it can be used in statistical framework for hypothesis testing and inference [29]. We implemented the analysis in the SPM toolbox Multivariate Methods for fMRI (https ://github.com/LREN-CHUV/MLM).

The steps of the following analysis are described in more detail in our recent publication [23] and will be given shortly here:
(1) In the first step, we conducted an MLM analysis for each participant (Fig. 1, Individual Level MLM) identifying the brain patterns that describe most of the changes in the BOLD signal and that are most correlated with the three experimental conditions (Pos, $\mathrm{Neg}$, and $\mathrm{Neu}$ ). The experimental paradigm, was represented in a design matrix $X$, including the three types of stimuli (Pos, Neg and Neu) and the nuisance covariates (the six realignment parameters). For each subject $i(i=1 . . s)$ we computed the principal components of $Z_{i}$ the complex normalized correlation matrix of

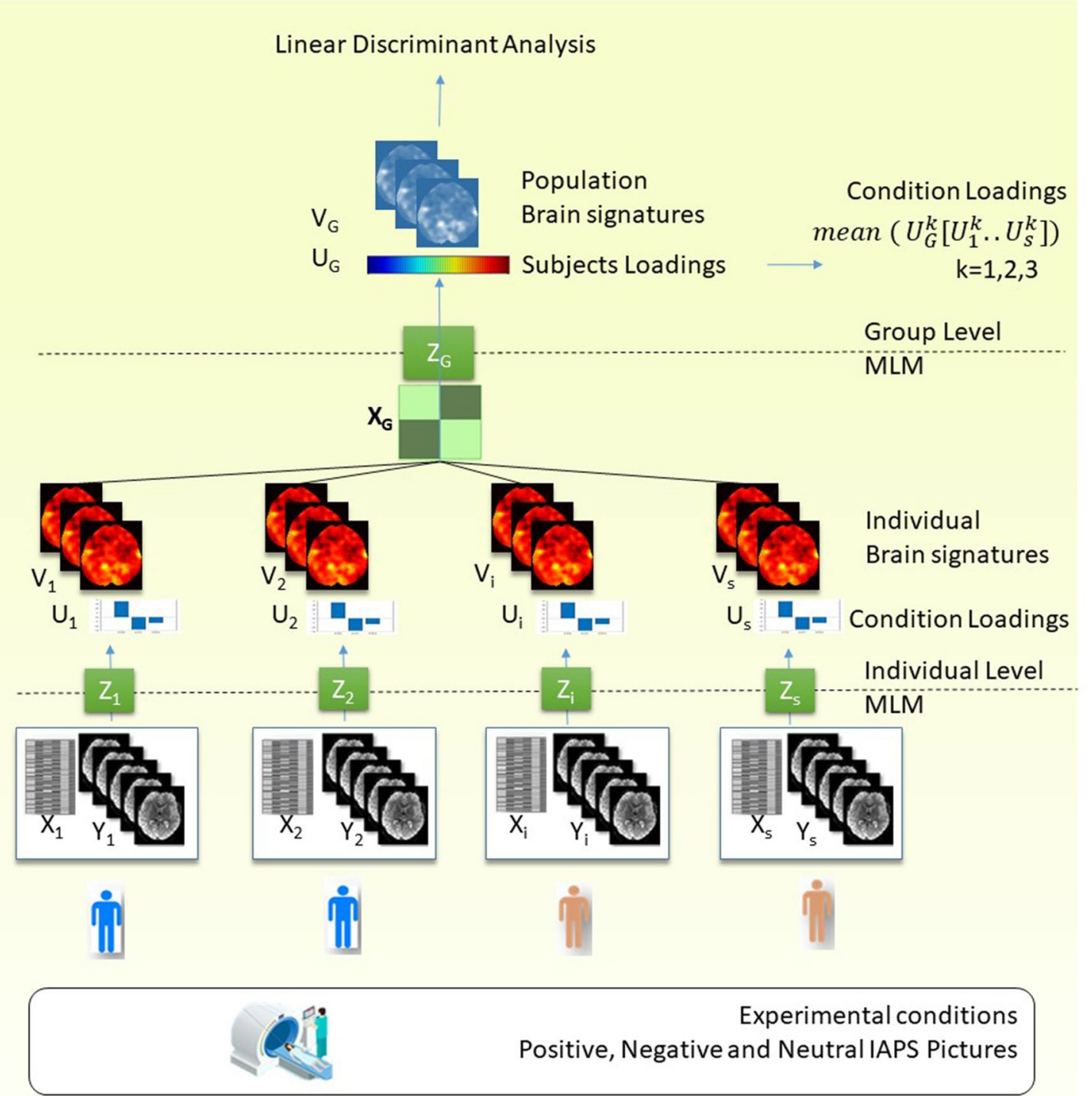

Fig. 1 Procedure steps: (1) Individual MLM: MLM decomposed covariance matrix between the functional data and the design matrix containing the experimental conditions into three components (or condition loadings) and three brain signatures (or eigenimages). (2) Group MLM: MLM analysis is performed on the covariance matrix between individual level eigenimages and the design matrix encod- ing the diagnostic label (healthy vs depressed) and the confounding variables (age, sex). This resulted in group level brain signatures (or eigenimages) and the subject loadings that discriminate between the groups. (3) We used a linear discriminant analysis to assess the predictive power of the brain signature 
the design matrix $X_{i}$ (time by covariates) and the data matrix $Y_{i}$ (time by voxel). Each matrix $Z_{i}$ is decomposed into model parameters eigenvectors $U_{i}$ and spatial eigenvectors $V_{i}$. The spatial eigenvectors are referred as eigenimages.

(2) The second step summarized the information from the individual MLMs by building a second level factorial design in SPM on each of the three eigenimages. The population level confounds age and gender were removed [30, 31]. We then performed a second MLM analysis (Fig. 1, Group Level MLM). At this level we build the matrix $Z_{G}$, constructed as a complex normalized correlation matrix of the design matrix $X_{G}$ (subject by covariates) and $Y_{G}=\left[U_{1}, U_{2}, \ldots, U_{s}\right]$, the eigenimages (active conditions by voxel) calculated during the previous step. The matrix $Z_{G}$ is then decomposed to find eigenvectors $U_{G}$ and $V_{G}$. The $V_{G}$ identify the most consistent brain pattern across individuals in terms of variance explained. The $U_{G}$, the subject loadings quantify individual differences i.e. the contribution of each subject to the main brain pattern.

(3) In the final step, we applied a linear discriminant analysis classifier (LDA in Statistics and Machine learning toolbox, Matlab R2020a) on each of the three subject loadings to assess whether the brain signatures can differentiate between the depressed patients and the healthy controls. Accuracy of classification was obtained using $\mathrm{k}$-fold $(\mathrm{k}=2)$ cross-validation technique.

All the steps of the analysis are schematically explained in Fig. 1 where it can be seen that we used multivariate method at individual and group levels in order to compute the relevant brain signatures.

\subsection{Statistical Analysis}

In this study we used SPSS 22.0 running on Windows for the statistical analysis of the demographic and clinical characteristics of the participants. Continuous variables were tested with Student's t-test and categorical ones with Chi-square test. The chosen level of significance was $p<0.05$ for all tests.

\section{Results}

\subsection{Demographic and Clinical Characteristics}

The depressed patients and the matched healthy controls did not differ in their age, sex and education level. Expectedly, significantly higher total scores on the Montgomery-Åsberg Depression Rating Scale were characteristic for the depressed participants. The two patient subgroups were not significantly different in neither of the demographic and clinical characteristics explored (see Table 1).

\subsection{MLM Results}

Step 1: The individual level MLMs demonstrated a consistent profile across all participants with the first component corresponding to positive loading for the Neu condition and negative loadings for both the Pos and the Neg
Table 1 Demographic and clinical characteristics of the participants

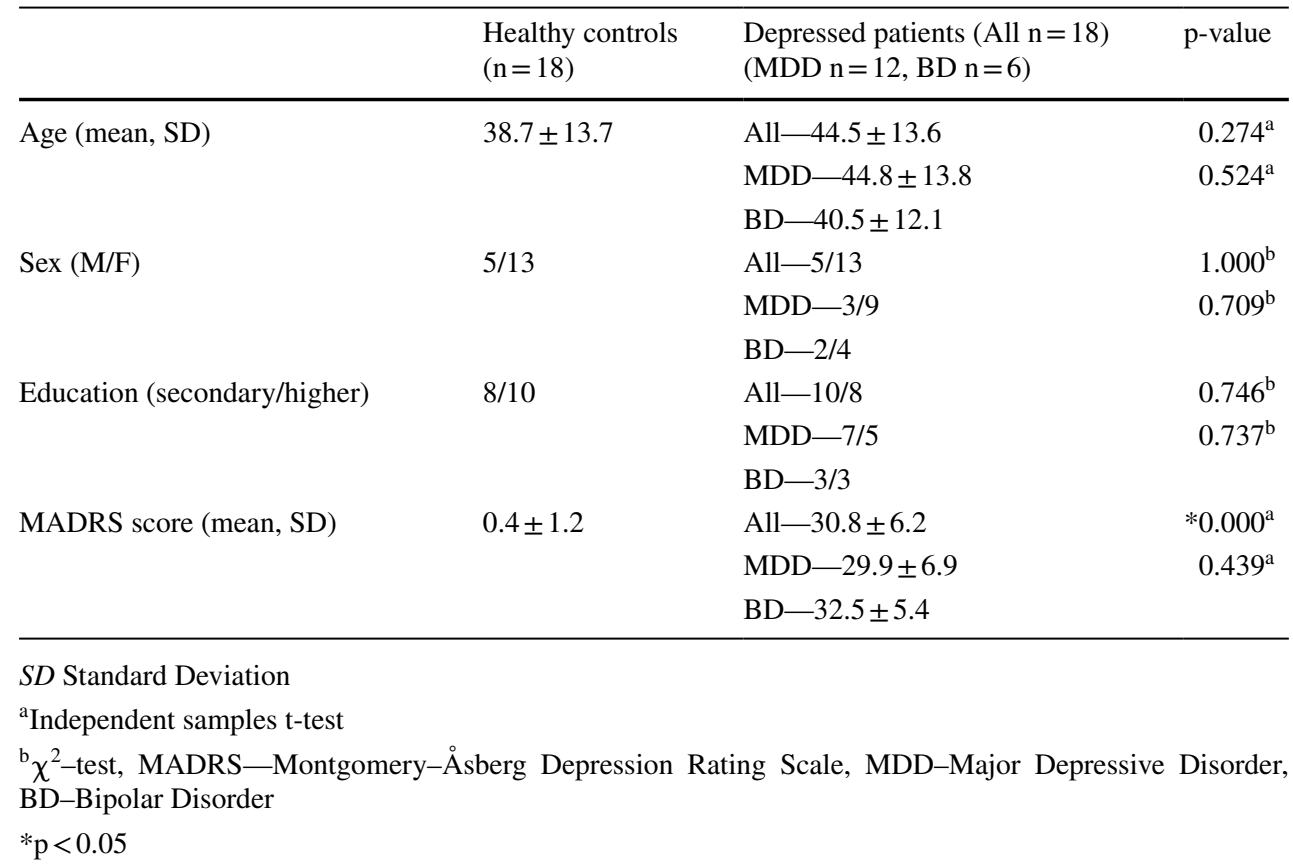


pictures. The second component, had negative loadings less pronounced for the Pos and more for the Neg combined with positive Neu loadings. The last component was characterized by a positive loading for the Neg condition and negative loadings for Pos and Neu.
Step 2: The group level MLM captured the three brain signatures that summarized the variabilities of the individual brain patterns (see Fig. 2). The first of them had a positive pattern spanning across different parts of the frontal lobes (superior and middle frontal gyrus, orbitofrontal cortex, frontal operculum), motor cortex, occipital and some
Fig. 2 MLM results: subject and Brain projections across all participants. Panels A, B, and $\mathrm{C}$ show the subject loadings for the first, second and third components, the corresponding Eigen-images projected on a 3D brain and the condition loadings. Solid blue line indicates the observed subjects' loadings, and the dotted line represents the predicted ones. The brain signatures show the statistical association (T-Test) between the subjects' loadings and the value at each voxel. The strength of this correlation is for illustration purposes. The group condition loadings are the averaged loadings of each subject calculated at the individual level MLM and weighted by the subject loading obtained at the group level MLM

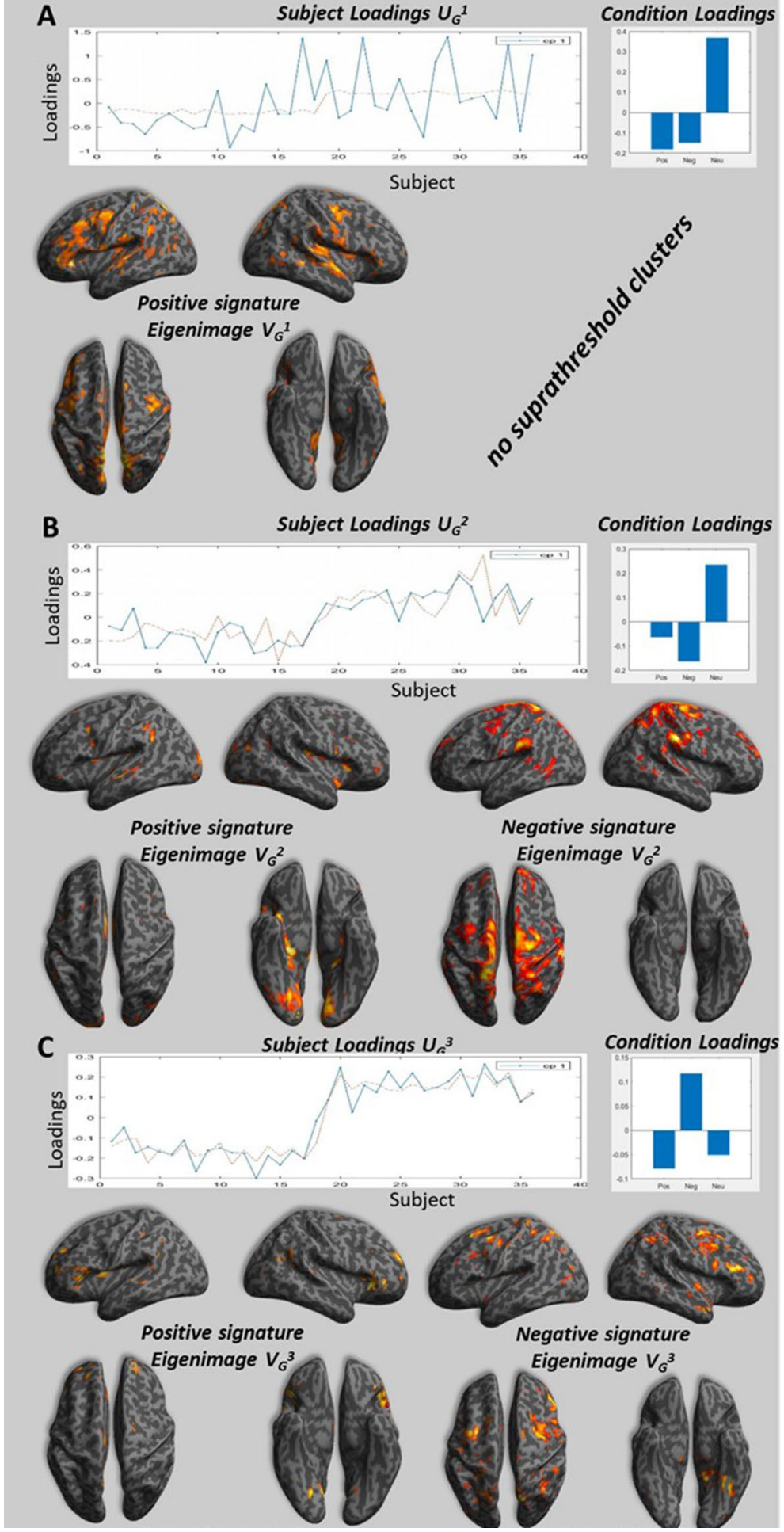


subcortical structures such as thalamus and putamen. The second signature demonstrated much more concise clusters in calcarine cortex, cuneus, precuneus, insular cortex, hippocampus and parahippocampal gyrus for the positive pattern and in supramarginal gyrus, motor cortices, ventromedial prefrontal areas extending to the anterior cingulate cortex (ACC), and planum temporale for the negative one. The third signatures' positive pattern encompassed parts of the ventrolateral and the dorsolateral PFC, lingual gyrus, as well as smaller cluster in supramarginal gyrus. However, the negative pattern demonstrated smaller clusters in parietotemporal (superior parietal lobule, angular and middle temporal gyrus) and frontal regions (middle frontal gyrus and dorsal anterior cingulate).

Step 3: The cross-validated accuracy (twofold cross validation and repeated 100 times to estimate the medians and 25th and 75th percentiles of its distribution.) of the linear discriminant analysis on the subjects' loadings for the three brain signatures was measured. The median accuracy was 0.67 for the first brain signature, 0.92 for the second and 0.98 for the third signature.

\section{Discussion}

The results of this study demonstrated that by using the methods of multivariate analysis (namely MLM) on emotional task related fMRI data we were able to derive meaningful brain signatures on a group level that reflected the underlying regional activity. Those consistent patterns of brain activation were further used to achieve clinically significant levels of accurate discrimination between the two groups of participants (spanning from above 67 to 98\%) which was not possible when applying classical mass univariate analysis with stringent criteria of inference (e.g. FWE correction for multiple comparisons) as demonstrated in a previous publication on the same data [20].

The brain signatures that were produced by the MLM analysis clearly encompassed regions related to the emotional visual task. The first of them represented broad activations in areas of the frontal lobes (superior and middle frontal gyrus, orbitofrontal cortex, frontal operculum), motor cortex, occipital regions and some subcortical structures such as thalamus and putamen. Some of those regions are related to the behavioural component of the task (e.g. motor cortex-button press) but most of them are implicated in the processing of visual stimuli with different emotional valence [32-34]. Since this signature yielded the least significant distinction between the two groups, we will focus more on the other two.

The second brain signature demonstrated more concise clusters with a positive pattern in calcarine cortex, cuneus, precuneus, insular cortex, hippocampus and parahippocampal gyrus and a negative pattern in supramarginal gyrus, motor cortices, ventromedial prefrontal areas extending to the anterior cingulate cortex (ACC), and planum temporale. The positive pattern relates to areas of activation that contribute to the distinction between the groups being more pronounced in the patients, while the negative pattern demonstrates the opposite - higher activation in the control group. The experimental condition loadings for this brain signature were reflecting positive and negative loadings for the Neu and Neg conditions respectively (see Fig. 2).

The activation of cuneus, insula and hippocampus might be linked to the performance part of the task that was used for monitoring participant's attention as those brain areas have been found active in similar functional paradigms [35]. We might speculate that the patients recruited in a higher extent those areas as it is well known that impairment of attention, memory and decision making is a core feature of depression [36]. In addition, both functional and structural MRI studies implicate the role of the hippocampus in depression with findings of reduced volume, disturbed activity and connectivity that can be seen even in unmedicated subjects and are linked to illness severity and treatment outcome [37-41].

The ventromedial PFC and the ACC have been suggested to play an important role in both emotion processing and pathophysiology of depression [42-44]. Moreover, depressed patients demonstrate distinct connectivity disturbances of specific subdivisions of the ACC with parts of the PFC [45]. The effective connectivity of the fronto-limbic circuit has been suggested as a predictor of response to antidepressants [46]. Most recent study suggests that stimulation of pre- or subgenual ACC might be an effective treatment for depression [47]. Our results of less activation of the abovementioned regions in depressed patients are in line with the cited literature.

The third signature reflects a positive loading for the Neg condition and a negative loading for the Pos pictures. It produced the highest level of discrimination accuracy of the classifier and we feel tempted to suggest an interpretation of this fact in line with the mainstream findings of more pronounced disturbances of the processing of negative stimuli (of various nature) in depression, e.g. the negative bias that is suggested as well to predict treatment outcome [48-51].

The positive pattern of this last brain signature encompassed parts of the ventrolateral and the dorsolateral PFC, lingual gyrus, as well as a smaller cluster in supramarginal gyrus on the left side which are more activated in the patient group. The functional area of the dorsolateral PFC is associated with executive functions-attention, working memory, planning, inhibition of response [52-55]. The disturbed activity of this region is associated with depression [43] and is targeted in transcranial magnetic 
stimulation treatment for resistant depression [56]. The supramarginal gyrus on the other hand is reported to be involved in the mechanisms of empathy [57]. The increased activation of this region in the patient group might be a reflection of the negative bias once again [4].

The negative pattern of the last brain signature demonstrated mainly smaller areas of activation in parietotemporal (superior parietal lobule/precuneus, angular and middle temporal gyrus) and frontal regions including clusters in middle frontal gyrus and dorsal anterior cingulate. This patter reflects more activation in healthy controls. Lisieacka et al. reported higher activations in control subjects located to the right precuneus/posterior cingulate cortex, right insula, left and right frontal operculum, left angular and left supramarginal gyrus during emotion processing [58].

In summary, the multivariate analysis of this highly dimensional data from a classical emotional pictures functional paradigm generated meaningful brain signatures that were not only convergent with the main findings within the field (in contrast with the negative findings of the previous analysis) but produced as well significant discriminative power as demonstrated by the final results of the classification.

This fact is even more important having in mind that the emotional stimuli are taken from the IAPS which on one hand is used for the investigation of the neural mechanisms underlying emotional processing in healthy individuals but has also been considered as a valuable instrument to assess affectivity in psychosis from a diagnostic and treatment perspective $[59,60]$. There is evidence that neutral scenes on IAPS cause significant over-arousal in patients with schizophrenia, which is attenuated during anti-psychotic treatment. There are also implications on the role of IAPS in the diagnostic process of affective and anxiety spectrum disorders [61].

We suggest that results of the present study along with other similar findings of our group [23] and other researchers focusing on the use of multivariate analysis will fuel the emerging paradigm shift from more conventional statistical analysis to the probably more appropriate for the functional neuroimaging field machine learning techniques.

However, the present study has a number of limitations that should be noted. Main concern is the relatively small sample size that might impair the generalizability of the results. The heterogeneity of the patient group in terms of diagnosis (major depressive disorder and bipolar disorder) has to be addressed too. We focused here on the syndromic presentation of depression and this is the reason to include two different diagnostic classes. In addition, the fact that the patients were receiving antidepressant medication prior to inclusion might have contributed to the results to some extent.

\section{Conclusion}

The present study adds to the growing body of evidence that multivariate linear models might be better suited for the analysis of task related fMRI paradigms where conventional mass univariate analysis might not yield significant results when more stringent criteria for statistical inference are employed, e.g. FWE correction for multiple comparisons. This method resulted in meaningful brain signatures that demonstrated significant potential for distinction between healthy and depressed subjects. Such findings will hopefully fuel the emerging paradigm shift from conventional statistical analysis to the probably more appropriate for the functional neuroimaging field machine learning techniques.

Author Contributions [DS]: Conceptualization; [SK, DS, FK, MS]: Methodology; [SK, AL]: Formal analysis and investigation; [SK, DS]: Writing-original draft preparation; [MS]: Writing-review and editing, [SK]: Resources; [FK]: Supervision.

Funding The research was supported by the budget of the Medical University of Plovdiv.

Data Availability Data related to this study is available upon request.

Code Availability https://github.com/LREN-CHUV/MLM.

\section{Compliance with Ethical Standards}

Conflicts of interest The authors declare that they have no conflicts of interest.

Ethical Approval The study was approved by local Research Ethics Committee at the Medical University of Plovdiv.

Consent to Participate Informed consent was obtained from all individual participants included in the study.

Consent for Publication The participants have consented to publication of the study results.

\section{References}

1. WHO (2011) Global burden of mental disorders and the need for a comprehensive, coordinated response from health and social sectors at the country level. WHO Geneva. https://apps.who.int/ iris/handle/10665/78898.

2. Hasler, G. (2010). Pathophysiology of depression: Do we have any solid evidence of interest to clinicians? World Psychiatry, 9(3), $155-161$.

3. Stoyanov, D., et al. (2018). Psychopathology assessment methods revisited: On translational cross-validation of clinical self-evaluation scale and fMRI. Frontiers in Psychiatry, 9, 21.

4. Leppanen, J. M. (2006). Emotional information processing in mood disorders: A review of behavioral and neuroimaging findings. Current Opinion in Psychiatry, 19(1), 34-39. 
5. Anand, A., et al. (2005). Activity and connectivity of brain mood regulating circuit in depression: A functional magnetic resonance study. Biological Psychiatry, 57(10), 1079-1088.

6. Fu, C. H., et al. (2004). Attenuation of the neural response to sad faces in major depression by antidepressant treatment: A prospective, event-related functional magnetic resonance imaging study. Archives of General Psychiatry, 61(9), 877-889.

7. Sheline, Y. I., et al. (2001). Increased amygdala response to masked emotional faces in depressed subjects resolves with antidepressant treatment: An fMRI study. Biological Psychiatry, 50(9), 651-658.

8. Canli, T., et al. (2005). Amygdala reactivity to emotional faces predicts improvement in major depression. NeuroReport, 16(12), 1267-1270.

9. Siegle, G. J., et al. (2007). Increased amygdala and decreased dorsolateral prefrontal BOLD responses in unipolar depression: Related and independent features. Biological Psychiatry, 61(2), 198-209.

10. Rosenblau, G., et al. (2012). Functional neuroanatomy of emotion processing in major depressive disorder is altered after successful antidepressant therapy. Journal of Psychopharmacology, 26(11), 1424-1433.

11. Borgwardt, S., \& Stoyanov, D. (2015). Emerging psychiatric neuroimaging findings: Translating research into clinical application. In D. Stoyanov (Ed.), Towards new philosophy of mental health, perspectives from neuroscience and humanities (pp. 35-40). Newcastle upon Tyne: Cambridge Scholars Publishing.

12. Borgwardt, S., et al. (2012). Why are psychiatric imaging methods clinically unreliable? Conclusions and practical guidelines for authors, editors and reviewers. Behavioural and Brain Function, $8,46$.

13. Fitzgerald, P. B., et al. (2008). A meta-analytic study of changes in brain activation in depression. Human Brain Mapping, 29(6), 683-695.

14. Diener, C., et al. (2012). A meta-analysis of neurofunctional imaging studies of emotion and cognition in major depression. Neuroimage, 61(3), 677-685.

15. Hagele, C., et al. (2016). Affective responses across psychiatric disorders-A dimensional approach. Neuroscience Letters, 623, 71-78.

16. Muller, V. I., et al. (2017). Altered brain activity in unipolar depression revisited: Meta-analyses of neuroimaging studies. JAMA Psychiatry, 74(1), 47-55.

17. Saggar, M., \& Uddin, L. Q. (2019). Pushing the boundaries of psychiatric neuroimaging to ground diagnosis in biology. ENeuro. https://doi.org/10.1523/ENEURO.0384-19.2019.

18. Kellmeyer, P. (2017). Ethical and legal implications of the methodological crisis in neuroimaging. Cambridge Quarterly of Healthcare Ethics, 26(4), 530-554.

19. Woo, C. W., Krishnan, A., \& Wager, T. D. (2014). Cluster-extent based thresholding in fMRI analyses: Pitfalls and recommendations. Neuroimage, 91, 412-419.

20. Kandilarova, S., et al. (2018). Application of functional magnetic resonance imaging in psychiatric clinical evaluation: Controversies and avenues. Journal of Evaluation in Clinical Practice, 24(4), 807-814

21. Lang, P., Bradley, M., \& Cuthbert, B. N. (2008). International affective picture system (IAPS): Affective ratings of pictures and instruction manual. Gainesville: University of Florida.

22. Stoyanov, D., et al. (2019). Cross-validation of paranoid-depressive scale and functional MRI: New paradigm for neuroscience informed clinical psychopathology. Frontiers in Psychiatry, 10, 711.

23. Stoyanov, D., et al. (2019). Cross-validation of functional MRI and paranoid-depressive scale: Results from multivariate analysis. Frontiers in Psychiatry, 10, 869.
24. Stoyanov, D. S. (2010). The endophenotype project and the validation theory: Integration of neurobiology and psychiatry. Folia Medica (Plovdiv), 52(1), 18-25.

25. Stoyanov, D., Machamer, P., \& Schafner, K. (2013). A fallacious forced choice: Cloninger and stoyanov, Machamer and Schafner are compatible. Phylosophy, Psychiatry \& Psychology, 20(3), 281-284.

26. Stoyanov, D., Borgwardt, S., \& Varga, S. (2014). The problem of translational validity across neuroscience and psychiatry. In P. Zachar (Ed.), Alternative perspectives on psychiatric validation (pp. 128-146). Oxford: Oxford University Press.

27. Sheehan, D. V., et al. (1998). The Mini-International Neuropsychiatric Interview (M.I.N.I.): The development and validation of a structured diagnostic psychiatric interview for DSM-IV and ICD-10. Journal of Clinical Psychiatry., 20, 22-33.

28. Montgomery, S. A., \& Asberg, M. (1979). A new depression scale designed to be sensitive to change. British Journal of Psychiatry, 134, 382-389.

29. Kherif, F., et al. (2002). Multivariate model specification for fMRI data. Neuroimage, 16(4), 1068-1083.

30. Draganski, B., Kherif, F., \& Lutti, A. (2014). Computational anatomy for studying use-dependant brain plasticity. Frontiers in Human Neuroscience, 8, 380.

31. Draganski, B., Lutti, A., \& Kherif, F. (2013). Impact of brain aging and neurodegeneration on cognition: Evidence from MRI. Current Opinion in Neurology, 26(6), 640-645.

32. Phan, K. L., et al. (2002). Functional neuroanatomy of emotion: A meta-analysis of emotion activation studies in PET and fMRI. Neuroimage, 16(2), 331-348.

33. Lang, P. J., et al. (1998). Emotional arousal and activation of the visual cortex: An fMRI analysis. Psychophysiology, 35(2), 199-210.

34. Phelps, E. A. (2004). Human emotion and memory: Interactions of the amygdala and hippocampal complex. Current Opinion in Neurobiology, 14(2), 198-202.

35. Parker, J. G., Zalusky, E. J., \& Kirbas, C. (2014). Functional MRI mapping of visual function and selective attention for performance assessment and presurgical planning using conjunctive visual search. Brain Behavior, 4(2), 227-237.

36. Gualtieri, C. T., \& Morgan, D. W. (2008). The frequency of cognitive impairment in patients with anxiety, depression, and bipolar disorder: An unaccounted source of variance in clinical trials. Journal of Clinical Psychiatry, 69(7), 1122-1130.

37. Guo, H., et al. (2014). Resting-state functional connectivity abnormalities in first-onset unmedicated depression. Neural Regeneration Research, 9(2), 153-163.

38. Seminowicz, D. A., et al. (2004). Limbic-frontal circuitry in major depression: A path modeling metanalysis. Neuroimage, 22(1), 409-418.

39. Brown, E. S., et al. (2014). Association of depressive symptoms with hippocampal volume in 1936 adults. Neuropsychopharmacology, 39(3), 770-779.

40. Arnone, D., et al. (2012). Magnetic resonance imaging studies in unipolar depression: Systematic review and meta-regression analyses. European Neuropsychopharmacology, 22(1), 1-16.

41. Frodl, T., et al. (2008). Effect of hippocampal and amygdala volumes on clinical outcomes in major depression: A 3-year prospective magnetic resonance imaging study. Journal of Psychiatry and Neuroscience, 33(5), 423-430.

42. Myers-Schulz, B., \& Koenigs, M. (2012). Functional anatomy of ventromedial prefrontal cortex: Implications for mood and anxiety disorders. Molecular Psychiatry, 17(2), 132-141.

43. Koenigs, M., \& Grafman, J. (2009). The functional neuroanatomy of depression: Distinct roles for ventromedial and dorsolateral prefrontal cortex. Behavioural Brain Research, 201(2), 239-243. 
44. Drevets, W. C., Price, J. L., \& Furey, M. L. (2008). Brain structural and functional abnormalities in mood disorders: Implications for neurocircuitry models of depression. Brain Structure and Function, 213(1-2), 93-118.

45. Yoshimura, S., et al. (2010). Rostral anterior cingulate cortex activity mediates the relationship between the depressive symptoms and the medial prefrontal cortex activity. Journal of Affective Disorders, 122(1-2), 76-85.

46. Vai, B., et al. (2016). Fronto-limbic effective connectivity as possible predictor of antidepressant response to SSRI administration. European Neuropsychopharmacology, 26(12), 2000-2010.

47. Jing, Y., et al. (2020). Pregenual or subgenual anterior cingulate cortex as potential effective region for brain stimulation of depression. Brain Behavior, 10(4), e01591.

48. Jaworska, N., et al. (2015). A review of fMRI studies during visual emotive processing in major depressive disorder. World Journal of Biological Psychiatry, 16(7), 448-471.

49. Heller, A. S., et al. (2013). Increased prefrontal cortex activity during negative emotion regulation as a predictor of depression symptom severity trajectory over 6 months. JAMA Psychiatry, 70(11), 1181-1189.

50. Gotlib, I. H., et al. (2004). Attentional biases for negative interpersonal stimuli in clinical depression. Journal of Abnormal Psychology, 113(1), 121-135.

51. Groenewold, N. A., et al. (2013). Emotional valence modulates brain functional abnormalities in depression: Evidence from a meta-analysis of fMRI studies. Neuroscience and Biobehavioral Reviews, 37(2), 152-163.

52. Johnson, J. A., Strafella, A. P., \& Zatorre, R. J. (2007). The role of the dorsolateral prefrontal cortex in bimodal divided attention: Two transcranial magnetic stimulation studies. Journal of Cognitive Neuroscience, 19(6), 907-920.
53. Kaller, C. P., et al. (2011). Dissociable contributions of left and right dorsolateral prefrontal cortex in planning. Cerebral Cortex, 21(2), 307-317.

54. Mars, R. B., \& Grol, M. J. (2007). Dorsolateral prefrontal cortex, working memory, and prospective coding for action. Journal of Neuroscience, 27(8), 1801-1802.

55. Ridderinkhof, K. R., et al. (2004). Neurocognitive mechanisms of cognitive control: the role of prefrontal cortex in action selection, response inhibition, performance monitoring, and reward-based learning. Brain and Cognition, 56(2), 129-140.

56. Pascual-Leone, A., et al. (1996). Rapid-rate transcranial magnetic stimulation of left dorsolateral prefrontal cortex in drug-resistant depression. The Lancet, 348(9022), 233-237.

57. Silani, G., et al. (2013). Right supramarginal gyrus is crucial to overcome emotional egocentricity bias in social judgments. Journal of Neuroscience, 33(39), 15466-15476.

58. Lisiecka, D. M., et al. (2013). Recruitment of the left hemispheric emotional attention neural network in risk for and protection from depression. Journal of Psychiatry and Neuroscience, 38(2), $117-128$.

59. Haralanova, E., et al. (2012). Subjective emotional over-arousal to neutral social scenes in paranoid schizophrenia. European Archives of Psychiatry and Clinical Neuroscience, 262(1), 59-68.

60. Haralanov, S., et al. (2015). Subjective emotional over-arousal to neutral social scenes in paranoid schizophrenia psychosis is significantly reduced by effective antipsychotic pharmacotherapy. American Journal of Psychology and Cognitive Science, 1(2), 48-60.

61. MacNamara, A., Kotov, R., \& Hajcak, G. (2016). Diagnostic and symptom-based predictors of emotional processing in generalized anxiety disorder and major depressive disorder: An event-related potential study. Cognitive Therapy and Research, 40(3), 275-289. 